\title{
Human pancreatic neuro-insular network in health and fatty infiltration
}

\author{
Shiue-Cheng Tang ${ }^{1,2,3} \cdot$ Luc Baeyens ${ }^{4,5}$ • Chia-Ning Shen ${ }^{6} \cdot$ Shih-Jung Peng ${ }^{1,2}$. \\ Hung-Jen Chien $^{2}$ • David W. Scheel ${ }^{4,5}$ - Chester E. Chamberlain ${ }^{4,5}$. \\ Michael S. German ${ }^{4,5,7}$
}

Received: 23 February 2017 / Accepted: 4 July 2017 /Published online: 29 August 2017

(C) Springer-Verlag GmbH Germany 2017

\begin{abstract}
Aims/hypothesis Identification of a pancreatic neuro-insular network in mice suggests that a similar integration of islets and nerves may be present in the human pancreas. To characterise the neuro-insular network and the intra-pancreatic ganglia in a clinically related setting, we examined human pancreases in health and with fatty infiltration via 3-dimensional (3D) histology and compared the human pancreatic microenvironment with its counterpart in mice.

Methods Human pancreatic specimens from individuals with normal BMI, high BMI $(\geq 25)$ and type 2 diabetes were used to
\end{abstract}

Shiue-Cheng Tang, Luc Baeyens and Michael S. German contributed equally to this work.

Electronic supplementary material The online version of this article (https://doi.org/10.1007/s00125-017-4409-x) contains peer-reviewed but unedited supplementary material, which is available to authorised users.

Shiue-Cheng Tang

sctang@life.nthu.edu.tw

1 Connectomics Research Center, National Tsing Hua University, Hsinchu, Taiwan

2 Institute of Biotechnology, National Tsing Hua University, Hsinchu, Taiwan

3 Department of Medical Science, National Tsing Hua University, 101, Sec. 2, Kuang Fu Rd, Hsinchu 30013, Taiwan

4 Eli and Edythe Broad Center of Regeneration Medicine and Stem Cell Research, University of California at San Francisco, San Francisco, CA, USA

5 Diabetes Center, University of California at San Francisco, San Francisco, CA, USA

6 Genomics Research Center, Academia Sinica, Taipei, Taiwan

7 Department of Medicine, University of California at San Francisco, San Francisco, CA, USA investigate the neuro-insular network. Transparent specimens were prepared by tissue clearing for transmitted light and deeptissue fluorescence imaging to simultaneously visualise infiltrated adipocytes, islets and neurovascular networks.

Results High-definition images of human islets reveal that both the sympathetic and parasympathetic nerves enter the islet core and reside in the immediate microenvironment of islet cells. Around the islets, the neuro-insular network is visualised with $3 \mathrm{D}$ histology to identify the intra-pancreatic ganglia (peri-lobular and intra-parenchymal ganglia) and the islet-ganglionic association. In humans, but not in mice, pancreatic fatty infiltration (BMI dependent) features adipocytes infiltrating into the parenchyma and accumulating in the perilobular space, in which the peri-lobular ganglia also reside. We identified the formation of adipose-ganglionic complexes in the peri-lobular space and enlargement of ganglia around adipocytes. In the specimen from the individual with type 2 diabetes, an increase in the number of nerve projections from the intra-parenchymal ganglia is associated with severe fatty infiltration.

Conclusions/interpretation We present new perspectives of human pancreas and islet innervation via 3D histology. Our results strongly suggest that fatty infiltration in the human pancreas creates a neurotrophic microenvironment and promotes remodelling of pancreatic innervation.

Keywords Adipocyte $\cdot$ Autonomic innervation · BMI · Fatty infiltration · Human islet $\cdot$ Obesity $\cdot$ Pancreatic ganglia . Sympathetic nerve $\cdot$ Type 2 diabetes

$\begin{array}{ll}\text { Abbreviations } \\ \text { 3D } & \text { 3-dimensional } \\ \text { TH } & \text { Tyrosine hydroxylase } \\ \text { vAChT } & \text { Vesicular acetylcholine transporter }\end{array}$




\section{Introduction}

Regulation of insulin and glucagon secretion from the islet beta and alpha cells is most important for glucose homeostasis. Physiologically, metabolites in the blood and stimulation from parasympathetic and sympathetic nerves work in concert to modulate islet hormone secretion [1-4]. For example, in humans the cephalic secretion of pancreatic polypeptide and insulin induced by food ingestion is largely attributed to autonomic activation [5-8]. In contrast, in severe hypoglycaemia (glucose level $\leq 1.94 \mathrm{mmol} / \mathrm{l}$ ) the activation of pancreatic sympathetic nerves contributes to an overall increase in glucagon secretion in dogs [9]. These functional links, as well as the neuro-insular network identified in mice [10], indicate an association between islets and the autonomic nervous system. The mouse neuro-insular network also implicates a similar neuro-insular association in the human pancreas.

At the organ level, human and mouse pancreases are functionally similar, but their parenchymal and islet arrangements are somewhat different. The human pancreas is a solid organ with intra-lobular islets closely surrounded by the exocrine acini, whereas the mouse pancreas is a collection of soft lobules with islets primarily residing in the inter-lobular space [11], in which the ganglia also reside to establish the neuroinsular network [10]. In the human pancreas, there have been no comprehensive studies to identify the location of the intrapancreatic ganglia or to depict the spatial arrangement of an islet-ganglionic association. This lack of information prompted us to apply 3-dimensional (3D) histology to human specimens to systematically characterise islets, islet neurovascular networks and islet-ganglionic associations.

To elucidate the intra-pancreatic ganglia and the neuroinsular network in a clinically related setting, we classified the degree of pancreatic fatty infiltration (or pancreatic steatosis [12-14]) in human specimens to assess the remodelling of the pancreatic microenvironment by infiltrated adipocytes. Clinically, pancreatic fatty infiltration is associated with obesity and type 2 diabetes [14-16]. Because insulin promotes adipogenesis, which converts pre-adipocytes (such as fibroblasts) to adipocytes $[17,18]$, the high concentration of insulin in the pancreas creates a favourable environment for the process. Furthermore, because insulin stimulates adipocyte proliferation [19], and adipocytes are known to release angiogenic and neurotrophic factors for neurovascular recruitment $[20,21]$, these reactive responses suggest that the pancreatic parenchyma and neurovascular networks are remodelled by the adipocytes, which could disturb the existing pancreatic environment.

To examine the neuro-insular network in health and fatty infiltration, we applied 3D histology with tissue clearing [10, $22,23]$ to acquire tissue maps and high-definition images of the human pancreas. Taking full advantage of the 3D image data, we also compared the human pancreatic microenvironment with its counterpart in mice, highlighting the differences and similarities between the two systems. Here we present the application of our imaging approach to the human pancreas and the morphological and quantitative analyses of the islet neurovascular networks and ganglionic population.

\section{Methods}

Human and mouse pancreatic specimens Human pancreases were obtained from cadaveric donors through the University of California, San Francisco Islet Isolation Core Facility. The study was approved by the Office of Ethics and Compliance, Human Research Protection Program. Specimens from eight non-diabetic individuals and one individual with type 2 diabetes were used in this research. Pancreatic tissue blocks (approximately $10 \times 10 \times 10 \mathrm{~mm}$ ) were fixed in $4 \%$ formaldehyde for 2 days and then washed in PBS for 4 days at $4^{\circ} \mathrm{C}$. Specimens were later sectioned to $350 \mu \mathrm{m}$ in thickness by vibratome and transferred to $0.1 \%$ paraformaldehyde for preservation at $4^{\circ} \mathrm{C}$. Table 1 lists the sex, age, BMI, $\mathrm{HbA}_{1 \mathrm{c}}$ level and cause of death for the nine donors. No exclusion criteria were set prior to the histological analysis. The analysis was performed without prior knowledge of donor's pancreatic microanatomy.

Mouse pancreatic specimens were derived from four wild-type $\mathrm{C} 57 \mathrm{BL} / 6$ (B6) mice at 8 weeks of age and three high-fat-fed B6 mice at 20 weeks of age (16 weeks on diet [24]). Animals were kept in a temperaturecontrolled and pathogen-free facility with a $12: 12 \mathrm{~h}$ light:dark cycle and ad libitum access to food and water. Blood vessels of the mouse pancreases were labelled through cardiac perfusion of the lectin-Alexa Fluor 488 conjugate (Invitrogen, Carlsbad, CA, USA) followed by $4 \%$ paraformaldehyde perfusion fixation [23]. Then the pancreases were harvested and post-fixed in $4 \%$ paraformaldehyde solution for $40 \mathrm{~min}$ at $15^{\circ} \mathrm{C}$. Vibratome sections of the fixed pancreases were prepared and the sections were transferred to $0.1 \%$ paraformaldehyde for preservation at $4^{\circ} \mathrm{C}$. The Institutional Animal Care and Use Committees at National Tsing Hua University approved all animal procedures.

Tissue labelling and microscopy The fixed specimens were immersed in $2 \%$ Triton X-100 solution for $2 \mathrm{~h}$ at $15^{\circ} \mathrm{C}$ for permeabilisation. Eight different primary antibodies were used, as per manufacturer's instructions to immunolabel the tissues following the protocol outlined below. The antibodies used were mouse anti-CD31 (endothelial marker; MS-353-S1, Thermo Scientific, Fremont, CA, USA), guinea pig antiinsulin (GTX27842, GeneTex, Irvine, CA, USA), rabbit antiglucagon (2810-1, Epitomics, Burlingame, CA, USA), mouse 
Table 1 Donor information

\begin{tabular}{llllll}
\hline Donor & Sex & Age (years) & BMI & $\begin{array}{l}\mathrm{HbA}_{1 \mathrm{c}} \% \\
(\mathrm{mmol} / \mathrm{mol})\end{array}$ & Cause of death \\
\hline 1 & Female & 46 & 19.8 & $4.6 \%(27)$ & Cerebrovascular/stroke \\
2 & Male & 46 & 23.4 & $5.4 \%(36)$ & Blunt trauma \\
3 & Male & 46 & 23.7 & $5.3 \%(34)$ & Head trauma \\
4 & Male & 47 & 25.0 & $4.9 \%(30)$ & Cerebrovascular/stroke \\
5 & Male & 52 & 27.5 & $5.7 \%(39)$ & Cerebrovascular/stroke \\
6 & Female & 55 & 29.4 & $4.6 \%(27)$ & Cerebrovascular/stroke \\
7 & Female & 47 & 30.5 & $3.5 \%(15)$ & Cerebrovascular/stroke \\
8 & Male & 33 & 34.0 & $5.9 \%(41)$ & Intraventricular haemorrhage \\
9 & Male & 53 & 29.6 & $13.3 \%(122)$ & Cerebrovascular/stroke \\
\hline
\end{tabular}

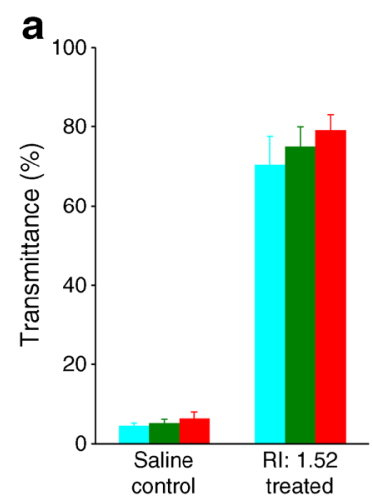

b
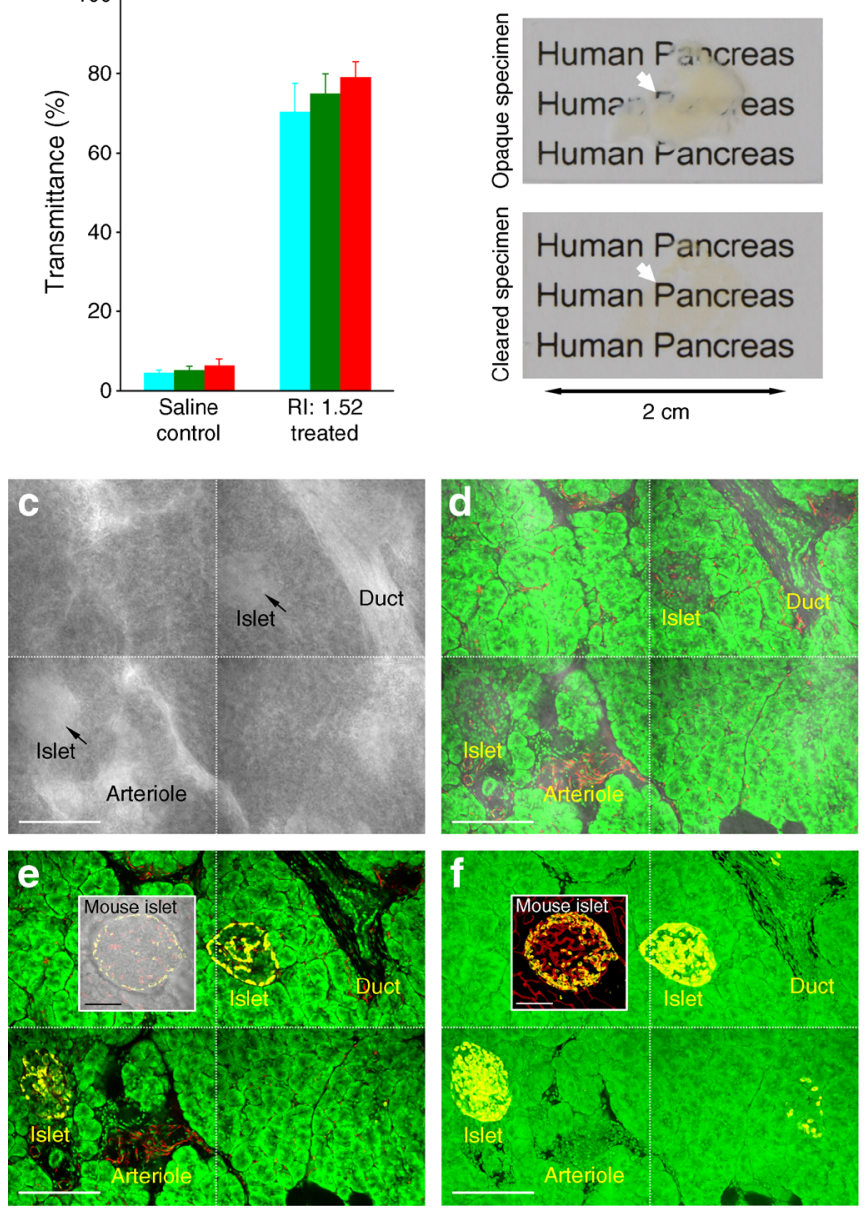

Fig. 1 Human pancreatic tissue clearing for 3D islet histology. (a, b) Increase in light transmission across human pancreatic specimens after tissue clearing. Specimens were immersed in a clearing solution with a refractive index (RI) at 1.52. In (a), transmittance was measured at $488 \mathrm{~nm}$ (cyan), $543 \mathrm{~nm}$ (green) and $633 \mathrm{~nm}$ (red). Data are presented as means \pm SD. $n=4$. Thickness of pancreatic section, $350 \mu \mathrm{m}$. (b) Side-byside comparison of opaque and cleared pancreases. (c-f) Gross view of islets in optically cleared pancreas. Islets in parenchyma shown by (c) transmitted light and (d) overlay of transmitted light and fluorescence signals. Glucagon staining (yellow) of islets shown as (e) 2D image and
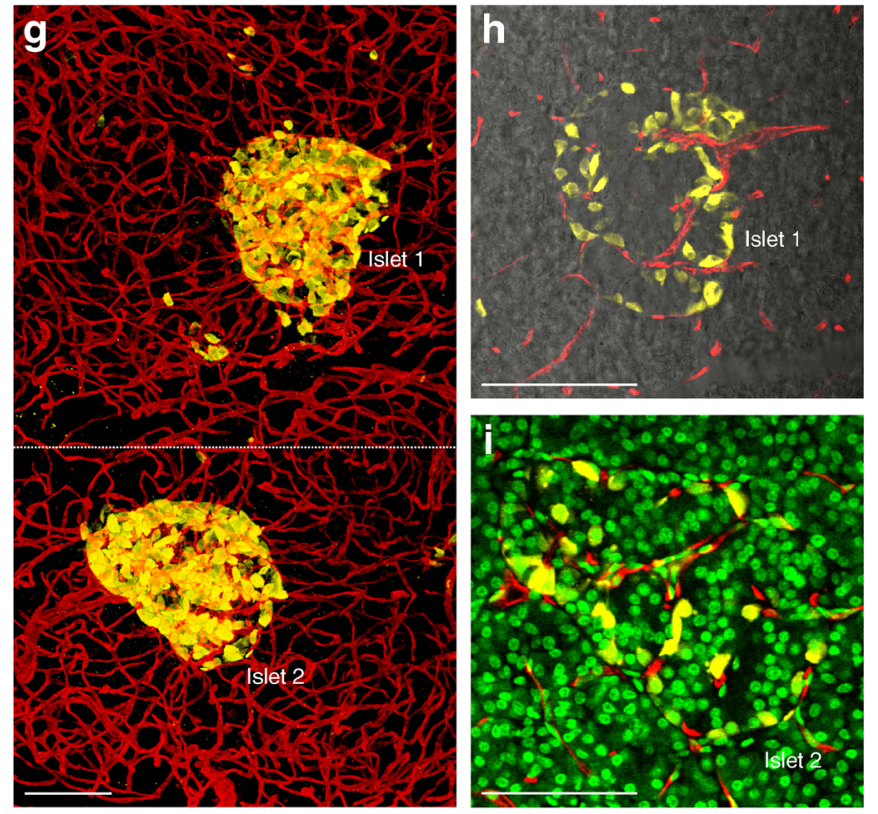

(f) 3D projection (depth, $50 \mu \mathrm{m}$ ). Red, vascular staining; green, nuclear staining and tissue autofluorescence. Tile scanning and image stitching were used to generate the tissue map. (g-i) Magnified examination of human islet. (g) Projection of glucagon-labelled islets and blood vessels (depth, $170 \mu \mathrm{m}$ ). (h, i) High-definition images of Islet 1 (transmitted light and fluorescence signals) and Islet 2 (fluorescence signals) in (g). Both (h) and (i) show that alpha cells are exclusively aligned along the microvessels, a unique feature of human islets [28]. Scale bars: main images, $200 \mu \mathrm{m}$; insets, $100 \mu \mathrm{m}$ 


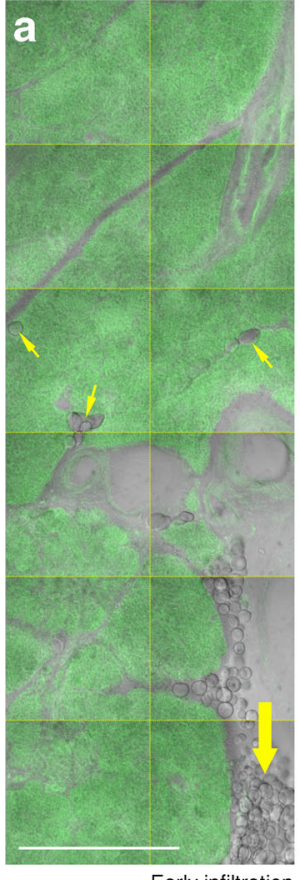

Early infiltration

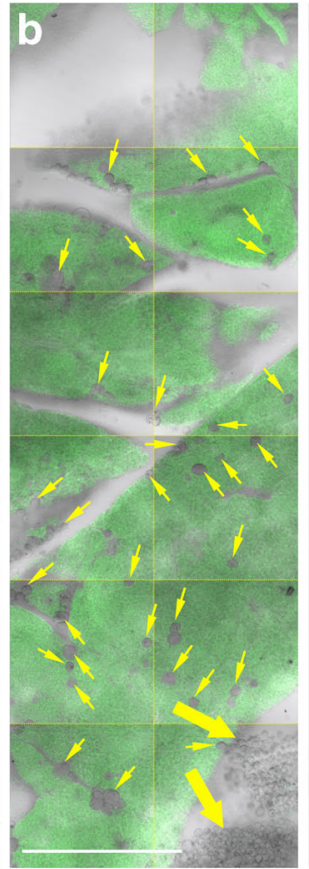

Moderate infiltration

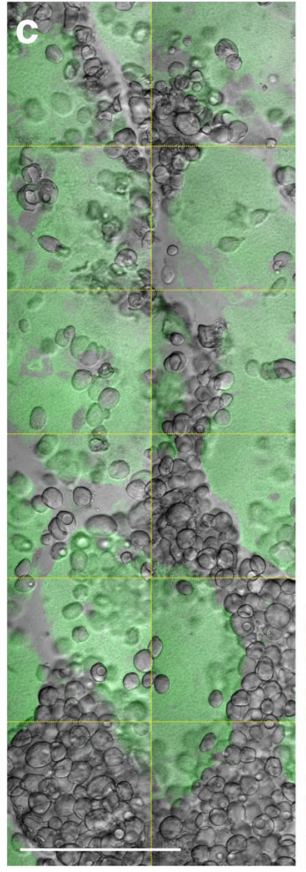

Severe infiltration

Fig. 2 Morphological and quantitative analyses of human pancreatic fatty infiltration. (a-d) Association of pancreatic fatty infiltration with BMI and metabolic conditions. Three levels of fatty infiltration, (a) early, (b) moderate and (c) severe, are linked with normal BMI, high BMI and type 2 diabetes (T2D), respectively (except for donor 8). Small and large arrows in $(\mathbf{a}, \mathbf{b})$ indicate the intra-parenchymal and peri-lobular adipocytes, respectively. Severe infiltration and hypertrophy of adipocytes are seen in the type 2 diabetic specimen (c). Scale bars, $1 \mathrm{~mm}$. In (d) (pixels

anti-glucagon (ab10988, Abcam, Cambridge, MA, USA), rabbit anti-tyrosine hydroxylase (TH, sympathetic marker; AB152, Millipore, Billerica, MA, USA), rabbit anti-vesicular acetylcholine transporter (vAChT, parasympathetic marker; 139103, Synaptic Systems, Goettingen, Germany), rabbit anti-PGP9.5 (neuronal marker; 2932-1, Epitomics) and rabbit anti-S100B (glial marker; ab52642, Abcam). Before applying the antibodies, tissue sections were rinsed in PBS. This was followed by a blocking step by incubating the tissue with a blocking buffer (2\% Triton X-100, 10\% normal goat serum, and $0.02 \%$ sodium azide in PBS). The primary antibody was then diluted in the dilution buffer $(1: 100,0.25 \%$ Triton X-100, $1 \%$ normal goat serum and $0.02 \%$ sodium azide in PBS) to replace the blocking buffer and incubated for 2 days at $15^{\circ} \mathrm{C}$.

Alexa Fluor 647, 546 and 488 conjugated secondary antibodies (raised in goat; 1:200, Invitrogen) were used in combination to reveal the immunostained structures. Propidium iodide or SYTO 16 staining was performed to reveal the nuclei. The labelled specimens were immersed in the optical clearing solution (RapiClear 1.52
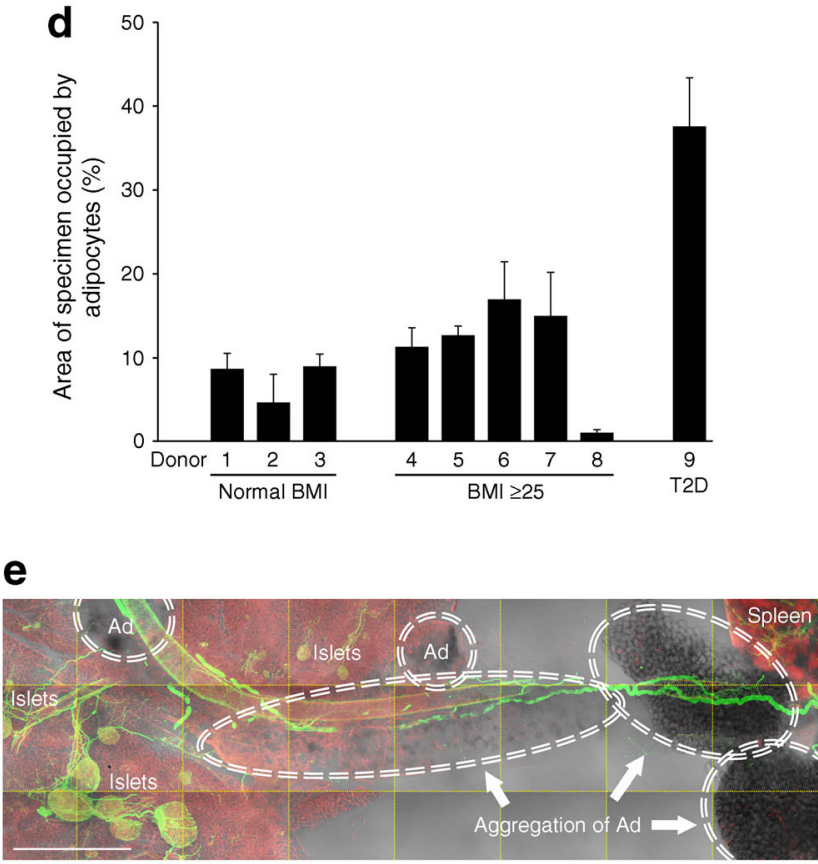

of adipocytes/overall pixels of specimen) $\times 100 \%$ was used to estimate the percentage of the specimen occupied by adipocytes. Transmitted light images of vibratome sections $\left(n \geq 5\right.$; each with an area $>40 \mathrm{~mm}^{2}$ ) were used in analysis. (e) Pancreatic fat attachment in mouse obesity. In the high-fat-fed mice (16 weeks on diet) [24], aggregation of adipocytes (Ad) is prominently seen around the pancreas and spleen, but there is minimal parenchymal infiltration. Red, vascular staining; green, neuronal marker PGP9.5. Projection depth, $350 \mu \mathrm{m}$. Scale bar, $1 \mathrm{~mm}$

solution, SunJin Lab, Hsinchu, Taiwan) before imaging via confocal microscopy [10, 23]. To quantify the tissue transparency, the percentage of light transmittance was measured with the microplate reader (SpectraMax M2e; Molecular Devices, Sunnyvale, CA, USA) [25].

Imaging of the tissue structure was performed with Zeiss LSM 510 Meta or LSM 800 confocal microscopes (Carl Zeiss, Jena, Germany) equipped with x10 Fluar (optical section, $10 \mu \mathrm{m}$; z-axis increment, $5 \mu \mathrm{m}), \times 25$ LD PlanApochromat (working distance, $570 \mu \mathrm{m}$; optical section, $5 \mu \mathrm{m}$; z-axis increment, $2.5 \mu \mathrm{m})$ and $\times 40 \mathrm{LD} C$ Apochromat (working distance, $620 \mu \mathrm{m}$; optical section, $3 \mu \mathrm{m}$; z-axis increment, $1.5 \mu \mathrm{m}$ ) lenses. Gross views of pancreatic lobules were acquired under $\times 10$ lenses via tile-scan mode with automatic image stitching. The laser-scanning process was operated under the multi-track scanning mode to acquire signals, including transmitted light signals. The transparent specimens were also examined with the Andor Dragonfly high-speed confocal imaging system (Andor Technology, Belfast, UK) to detect and confirm the pancreatic 


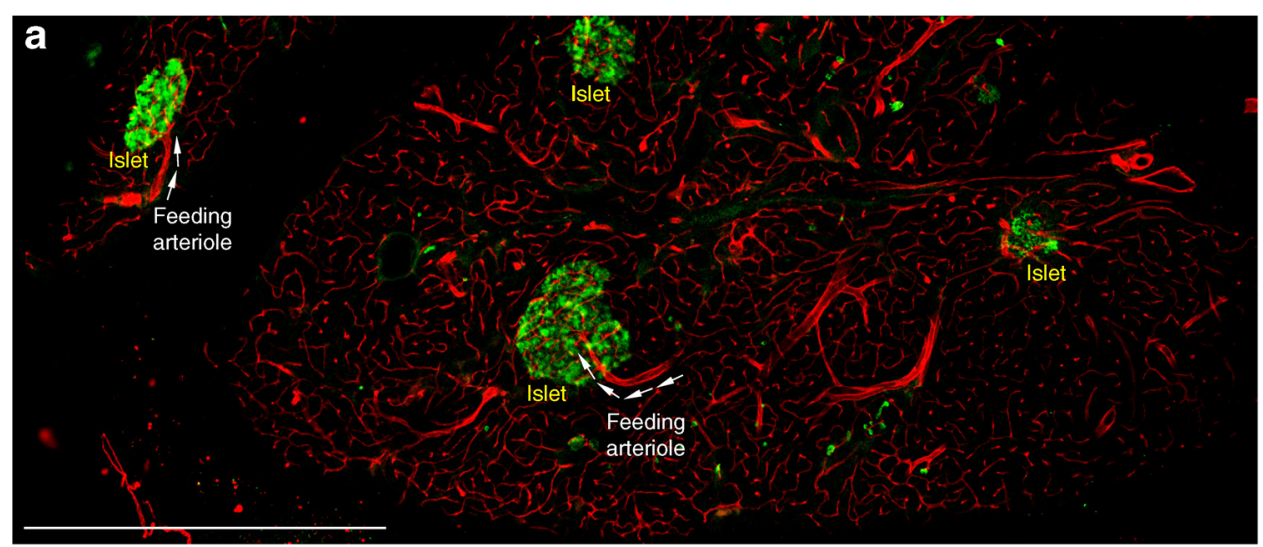

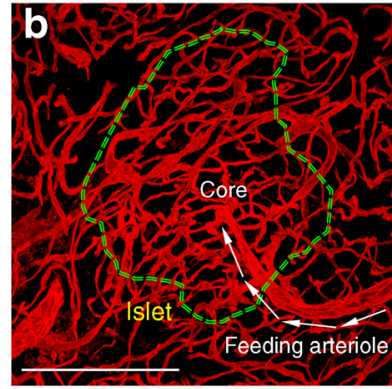

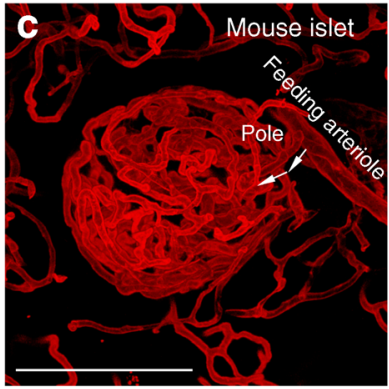

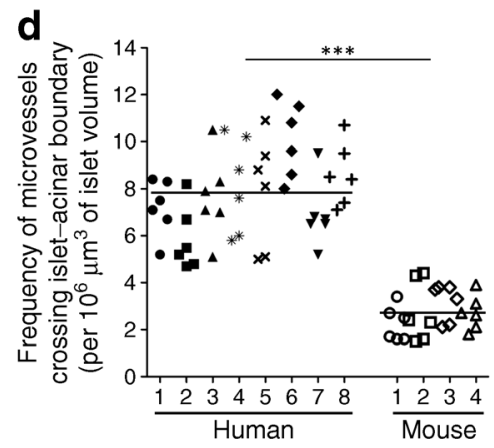

microvessels crossing the islet-acinar boundary in humans than in mice. Islets with a major axis $>200 \mu \mathrm{m}$ were selected for quantification. Horizontal lines indicate the mean of data points (human: 48 islets from eight donors; mouse: 24 islets from four mice; *** $p<0.001$; different shapes/symbols indicate different donors/mice). Note that the smaller islets and the scattered islet cells in the human pancreas may experience different microcirculatory patterns tissue networks (electronic supplementary material [ESM] Video 3).

Image processing and projection The Avizo 6.2 image reconstruction software (VSG, Burlington, MA, USA), Zen software (Carl Zeiss) and LSM 510 software (Carl Zeiss) were used for processing, projection and analysis of the confocal image stacks. Avizo's noise filtering algorithms were applied for background noise reduction. Signal tracing and feature extraction were performed by the Label Field function of Avizo to estimate the tissue volume via the occupied voxels [26]. Image stacks presented in ESM Videos were recorded using the Movie Maker function of Avizo with increase in display time in association with the depth of the optical section.

Statistical analysis The quantitative values are presented as means \pm SD or with the distribution of data points. Statistical differences were determined by the unpaired Student's $t$ test. Differences between groups were considered statistically significant when $p<0.05$.

\section{Results}

Tissue clearing of human pancreas for 3D islet histology Like all internal organs, the human pancreas strongly scatters light. Pancreatic tissue clearing (or optical clearing [27]) is thus essential to enable light penetration for optical imaging of the islet microenvironment. Figure $1 \mathrm{a}, \mathrm{b}$ shows the drastic increase in light transmission across the optically cleared human pancreases after immersion in the clearing solution (refractive index at 1.52). The increase in tissue transparency allows for simultaneous capture of transmitted light and fluorescence signals to identify the islets and surrounding acini (Fig. 1c-f). 

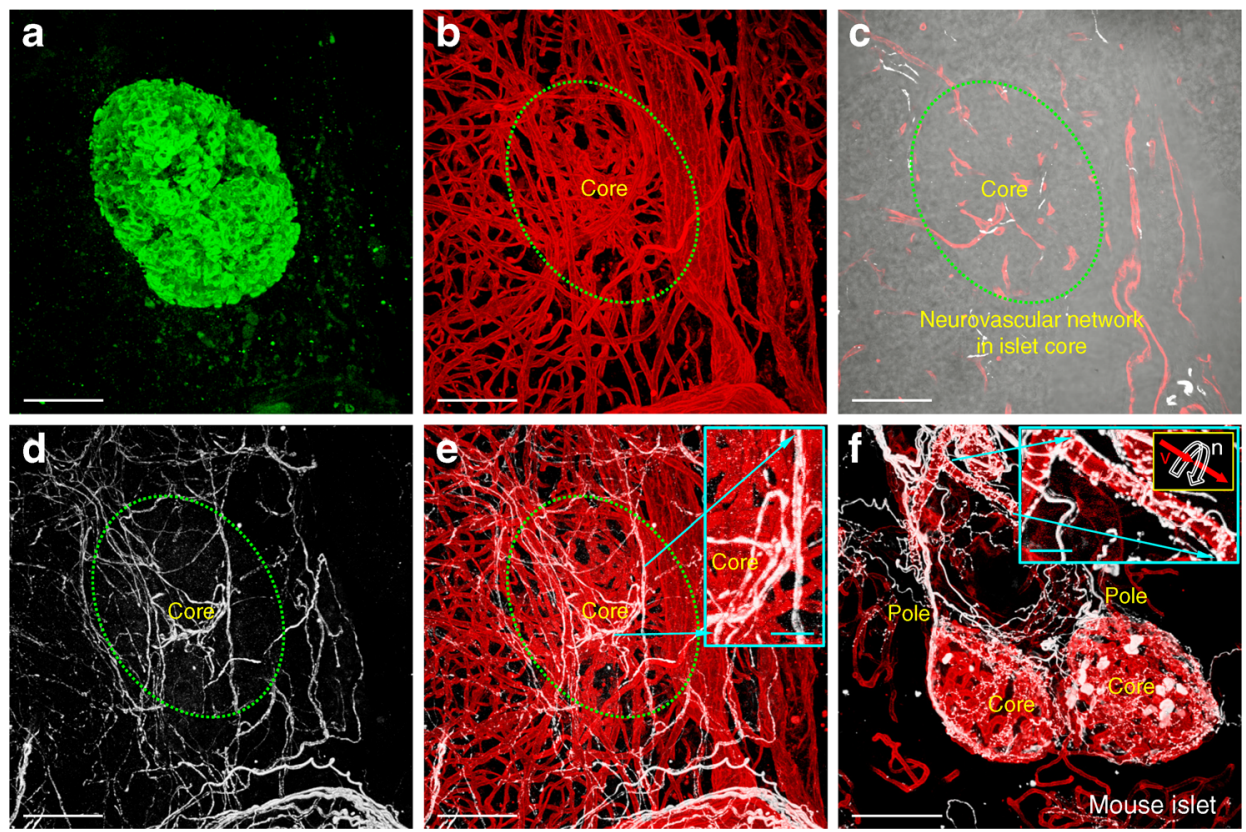

Fig. 4 Comparison of human and mouse islet neurovascular networks. (a-e) Human islet neurovascular network. 3D (a, b, d, e) and 2D (c) (overlay of transmitted light and fluorescence image) images of human islet microenvironment show that the sympathetic nerves follow microvessels into the islet core. Oval, islet boundary; green, insulin; red, vasculature; white, TH staining of sympathetic nerves. Inset in (e): magnified examination of perivascular sympathetic nerves. Projection depth, $350 \mu \mathrm{m}$. (f) Mouse islet sympathetic innervation. The projection shows that sympathetic nerves in the mouse pancreas also follow microvessels into the islet core. Magnified examination of the feeding arteriole (inset) shows that sympathetic nerves encircle the arteriole in extension (illustrated in the yellow box; $\mathrm{v}$, vessel; $\mathrm{n}$, nerve). This feature, however, is not seen in the human pancreas. White cell patches, THpositive endocrine cells. Scale bars: main image, $100 \mu \mathrm{m}$; inset, $20 \mu \mathrm{m}$
The pancreatic optical signals derived from this imaging approach show three features. First, after the clearing process, the endocrine islets are more transparent than the exocrine acini (Fig. 1c), which reflects the intrinsic difference between the endocrine and exocrine pancreas in composition and density. Second, tissue clearing allows the autofluorescence from the exocrine acini to be detected, serving as a counterstain to reveal the islet-acinar boundary and the pancreatic parenchyma (Fig. 1d, e). Third, glucagon staining shows that the human alpha cells reside in the islet core as well as the mantle (Fig. 1e-i; note that these alpha cells also align along the microvessels [28]), whereas the mouse alpha cells primarily reside in the mantle. The differences underscore the variation in islet cytoarchitecture across species [29].

Human pancreatic fatty infiltration Gross anatomy of the adult human pancreas often shows fat attachment at the surface. Microstructurally, the fats (or adipocytes) infiltrate into the pancreas, accumulating in the peri-lobular space and inside the parenchyma. Table 1 and Fig. $2 \mathrm{a}-\mathrm{d}$ show that the degree of fatty infiltration is associated with BMI, which is listed in ascending order in Table 1 (except for donor 9: type 2 diabetes, $\mathrm{HbA}_{1 \mathrm{c}} \geq 6.5 \%$ ). Analysis of the infiltrated adipocytes in the nine donors listed in Table 1 shows that normal BMI, high BMI $(\geq 25)$ and type 2 diabetes are linked with early $(<10 \%$ of fat area in specimen; pixels of adipocyte/pixels of specimen $\times$ $100 \%)$, moderate $(10-20 \%)$ and severe $(>30 \%)$ fatty infiltration, respectively (except for donor 8) (Fig. 2a-d). However, in mouse obesity (high-fat-fed mice, Fig. 2e), although the pancreatic fat attachment is prominent, fatty infiltration is rarely seen. In mice, adipocytes aggregate around the supporting blood vessels and nerves with minimal parenchymal infiltration. The result illustrates a drastic difference between mouse and human pancreases in adipocyte infiltration.

Human islet vasculature and neurovascular network Although human and mouse islets are similar in size, the patterns of human and mouse islet vascular networks are structurally different. In humans, examination of large islets $(>200 \mu \mathrm{m})$ shows that the islet feeding arteriole 

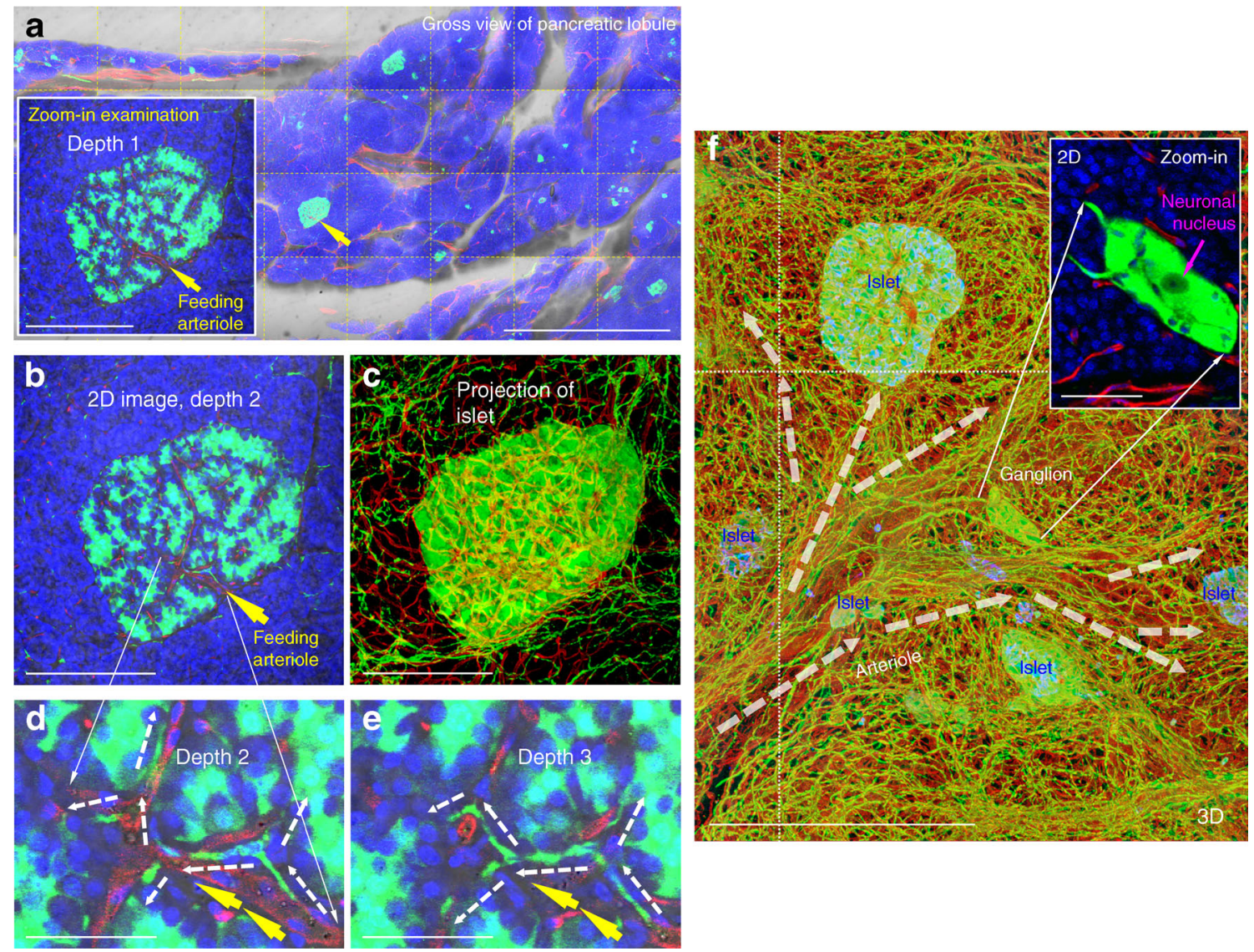

Fig. 5 Human pancreatic neuro-insular network. (a, b) Gross view and magnified examination of neuronal marker PGP9.5-labelled islets and nerves. Both human islets (neuroendocrine tissues) and nerves are labelled with PGP9.5 immunostaining (green). Arrow indicates an islet and the entry of its feeding arteriole. Red, CD31; blue, nuclei. Scale bars: (a) main image, $2 \mathrm{~mm}$; inset, $200 \mu \mathrm{m}$; (b) $200 \mu \mathrm{m}$. (c) In-depth projection of the PGP9.5-labelled islet and the associated nerves. Scale bar, $200 \mu \mathrm{m}$. Projection depth, $150 \mu \mathrm{m}$. (d, e) High-definition images of the neurovascular complex in an islet core taken at different focal depths.
Yellow arrows represent an extension of an arteriole; dashed white arrows represent an extension of PGP9.5-labelled nerves. Note that the PGP9.5labelled islet cells mask nerve fibre signals. Scale bars, $50 \mu \mathrm{m}$. (f) $3 \mathrm{D}$ panoramic projection of human pancreatic neuro-insular network. PGP9.5 staining (green) reveals a group of islets, nerve fibres and the intra-pancreatic ganglion (inset). Dashed arrows, arterioles; red, CD31; blue, insulin or nuclei (inset). Projection depth, $330 \mu \mathrm{m}$. Scale bars: main image, $500 \mu \mathrm{m}$; inset, $50 \mu \mathrm{m}$

fundamental difference in the islet microcirculation between the two species [31, 32].

Regarding the islet neurovascular network, the isletacinar vascular integration allows the nerves in parenchyma to follow microvessels entering the core from various directions. Using paired CD31 and TH (sympathetic marker) staining, we identified pancreatic perivascular sympathetic innervation (Fig. 4a-e). Examination of the islet microenvironment showed that the sympathetic nerves follow microvessels crossing the islet-acinar boundary to establish the pancreatic endocrine-exocrine integration.

Similarly in mice, the sympathetic nerves also follow microvessels in extension (Fig. 4f), reaching islets from their poles and entering the core. However, the mouse 

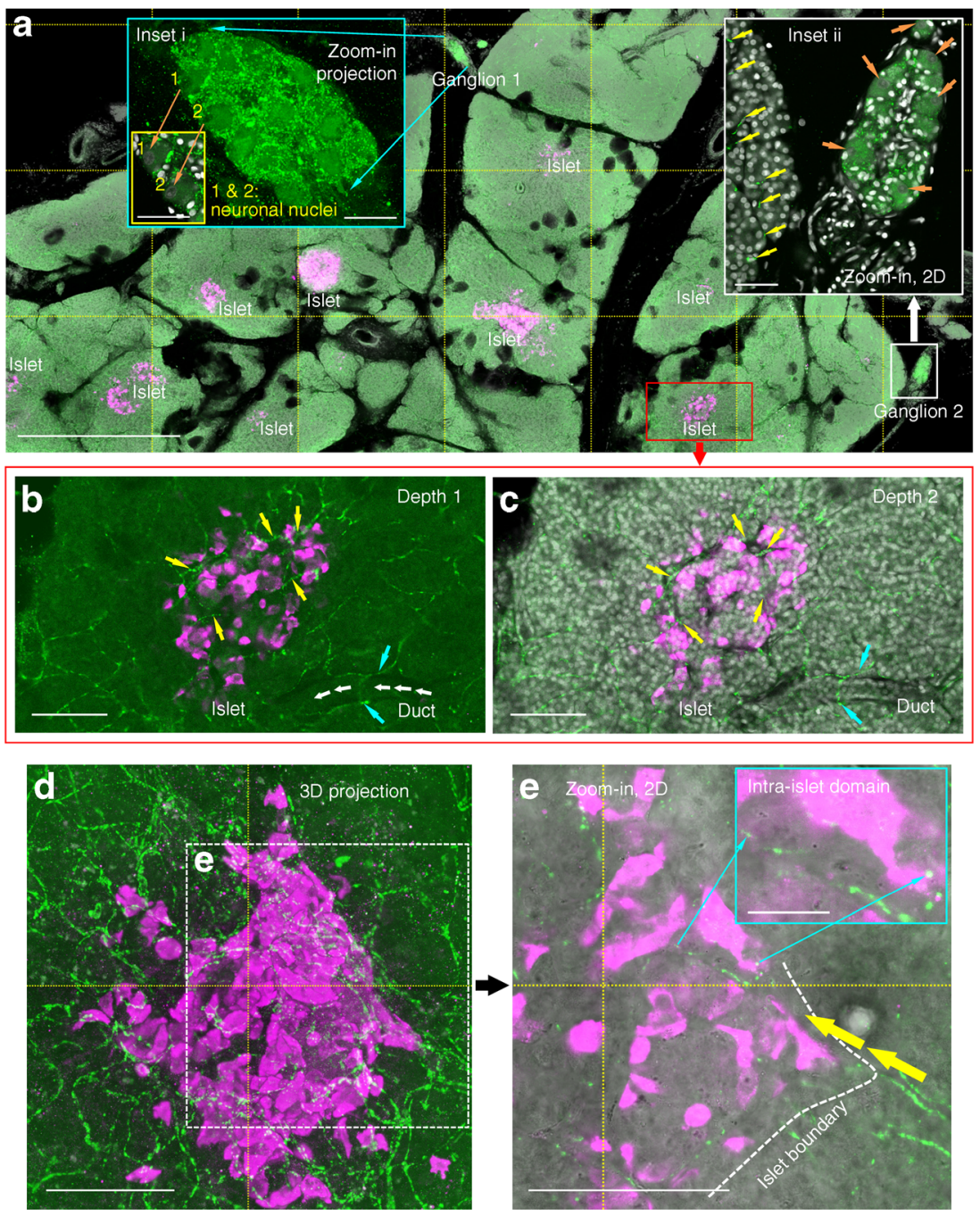

Fig. 6 Parasympathetic innervation of human intra-pancreatic ganglia and islets. (a) Pancreatic tissue map and magnified examination of intra-pancreatic ganglia. Islets are revealed by glucagon staining (magenta). Magnified examination of Ganglion 1 (inset i; scale bar, $50 \mu \mathrm{m}$ ) shows the parasympathetic marker vAChT (green)-labelled varicosities inside the ganglion. Projection depth, $18 \mu \mathrm{m}$. The varicosities (green dots) aggregate and reside at the boundary of the neuronal soma (yellow box, 2D image; scale bar, $50 \mu \mathrm{m}$; number 1 and 2 denote the dimly stained neuronal nuclei; white, nuclear staining). Inset ii (scale bar, $50 \mu \mathrm{m}$ ) shows the high-definition image of Ganglion 2 and the parasympathetic nerves in the parenchyma (yellow arrows). Orange arrows, neuronal nuclei. Scale bar for main image, $1 \mathrm{~mm}$. (b-e) Islet parasympathetic

innervation. (b) and (c) show the peri- and intra-islet $\mathrm{vAChT}^{+}$parasympathetic nerves at two focal depths (adjacent sections). Scale bars, $100 \mu \mathrm{m}$. Yellow arrows, intra-islet parasympathetic nerves; white arrows (in b), duct; cyan arrows, parasympathetic nerves around the duct. The vAChT signals were intentionally increased in (b) to visualise the parasympathetic innervation of acini and duct. $(\mathbf{d}, \mathbf{e})$ The $3 \mathrm{D}$ projection and the high-definition 2D image of islet parasympathetic innervation. Scale bars, $100 \mu \mathrm{m}$. The intra-islet domain is further enlarged in (e) (inset; scale bar, $20 \mu \mathrm{m}$ ) to identify the $\mathrm{vAChT}^{+}$nerves. Yellow arrows, extension of vAChT-labelled nerves; dashed line, islet boundary. ESM Video 2 provides a second example of the islet parasympathetic innervation with high definition

perivascular sympathetic nerves encircle the feeding arteriole in extension (inset in Fig. 4f; the structure has been suggested to control blood flow [33]); this encirclement is not observed in the human pancreas.

Human pancreatic neuro-insular network Islets have long been considered as neuroendocrine tissues [34]. This is

reflected by their endocrine cells which stain positively with the neuronal marker PGP9.5 (Fig. 5). Examination of a PGP9.5-labelled human pancreas shows that the islets are surrounded by a condensed network of nerve fibres, which reach the islet mantle and follow the microvessels into the core (Fig. 5a-e and ESM Video 1). Importantly, the nerve fibres extend from the islet and associate with the surrounding tissue structures, including nearby islets, microvessels and ganglia. 
Figure 5f illustrates a group of islets and the peri-islet microenvironment from a bird's eye view. This in-depth projection shows not only the islet-acinar neurovascular network but also the association of islets with the intra-pancreatic ganglia.

Parasympathetic innervation of intra-pancreatic ganglia and islets Although prior parasympathetic analysis of human islets has shown few, if any parasympathetic nerves penetrating the islet $[35,36]$, examination of the transparent human pancreas reveals that the endocrine islets, along with the intra-pancreatic ganglia and the exocrine pancreas (acini and ducts), are connected with the vAChT-labelled parasympathetic nerves (Fig. 6). Highdefinition images of the vAChT-labelled pancreas show that: (1) the parenchyma and the intra-pancreatic ganglia are both associated with parasympathetic nerves (Fig. 6a-c and ESM Video 2); (2) the intra-pancreatic ganglia are filled with the $\mathrm{vAChT}^{+}$varicosities (insets in Fig. $6 \mathrm{a})$, underscoring the parasympathetic regulation of pancreatic functions (the result also serves as the positive control of vAChT staining); (3) the parasympathetic nerves enter the islet core, implicating the immediate parasympathetic influence on the endocrine cells (Fig. 6b-e); (4) unlike the prior report stating that the alpha cells are $\mathrm{vAChT}^{+}$(i.e. presence of extensive $\mathrm{vAChT}^{+}$ cell patches in the islet) $[35,36]$, Fig. 6e and ESM Video 2 show that the glucagon-labelled alpha cells are largely vAChT negative; and (5) associated with the islet, the $\mathrm{vAChT}^{+}$varicosities connect in a $3 \mathrm{D}$ space continuum and neighbour the alpha cells (within $50 \mu \mathrm{m}$, ESM Video 2). The final two points allow us to trace and confirm the parasympathetic nerves in the islet core.

Intra-pancreatic ganglia and islet-ganglionic association in the human pancreas Next we used 3D histology with S100B (glial marker [37]) staining to characterise the intra-pancreatic ganglia and the glial network (Fig. 7ag). Two categories of intra-pancreatic ganglia are identified in the human pancreas: (1) the intra-parenchymal ganglia (Fig. 7d); and (2) the peri-lobular ganglia (Fig. $7 \mathrm{e}-\mathrm{g})$. In both subgroups, each ganglion features a swelling body with radiant projections and compartments that are S100B-negative, which appear to be the neuronal nuclei (dimly stained with nuclear dye). Furthermore, the supporting microvessels are seen in close contact with ganglia (Fig. 7e, f), indicating an intimate neurovascular association. ESM Video 3 presents a second example of the pancreatic glial network and the two subgroups of the intra-pancreatic ganglia.

Next, we used paired insulin and S100B staining to identify the islet-ganglionic association. Figure 7 h shows the panoramic projection of the glial network and its integration with the scattered islets. Upon magnification, high-definition images of the peri-islet ganglia confirm the islet-ganglionic association. The association is established via glial fibres extending from the peri-islet domain to the core (Fig. 7i-k and ESM Video 4) or direct islet-ganglionic contact (Fig. 71-r), in which both the islet and ganglion are at the edge of parenchyma.

Analysis of intra-pancreatic ganglia in health and fatty infiltration Pancreatic fatty infiltration features adipocytes infiltrating into the parenchyma and accumulating in the peri-lobular space (Fig. 2a-c), in which the perilobular ganglia also reside (Fig. 7a-g). This territorial overlap leads to the formation of adipose-ganglionic complexes, which are identified in the specimens from both the non-diabetic (Fig. 8a) and type 2 diabetic (Fig. 8b) donors. Morphologically, the adipose-ganglionic association features direct contacts between adipocytes and ganglia, and a prominent vascular supply to the complex. A side-by-side comparison between the normal peri-lobular ganglion (adipose-free) and the adiposeganglionic complex is presented in ESM Video 5. This video illustrates the remodelling of ganglionic microenvironment with fatty infiltration.

Quantification of the ganglia shows that the perilobular ganglia are significantly larger than the intraparenchymal ganglia in the specimens from the nondiabetic (both normal and high BMI) and type 2 diabetic donors (Fig. 8c). The results indicate the size, as well as positional, difference between the two subgroups of intra-pancreatic ganglia. Furthermore, examination of the peri-lobular ganglia shows that $23 \%$ of the ganglia $(n=65)$ are associated with adipocytes in the specimens from the non-diabetic donors. These ganglia are $47 \%$ larger than the peri-lobular ganglia away from the adipocytes (Fig. 8d). A similar increase in the size of peri-lobular ganglia $(63 \%)$ is identified in the type 2 diabetes specimen (donor 9).

It is worth noting that in the type 2 diabetes condition, both the peri-lobular and intra-parenchymal ganglia become larger in comparison with the other specimens (Fig. 8c), implicating a neurotrophic microenvironment in the type 2 diabetes pancreas.

In addition to the size difference between the perilobular and intra-parenchymal ganglia, we also identified that the peri-lobular ganglia have more nerve projections than the intra-parenchymal ganglia in the specimens from the non-diabetic donors (both normal and high BMI) (Fig. 8e). However, the difference becomes non-significant in the type 2 diabetes condition, in which a noticeable increase in projections from the 

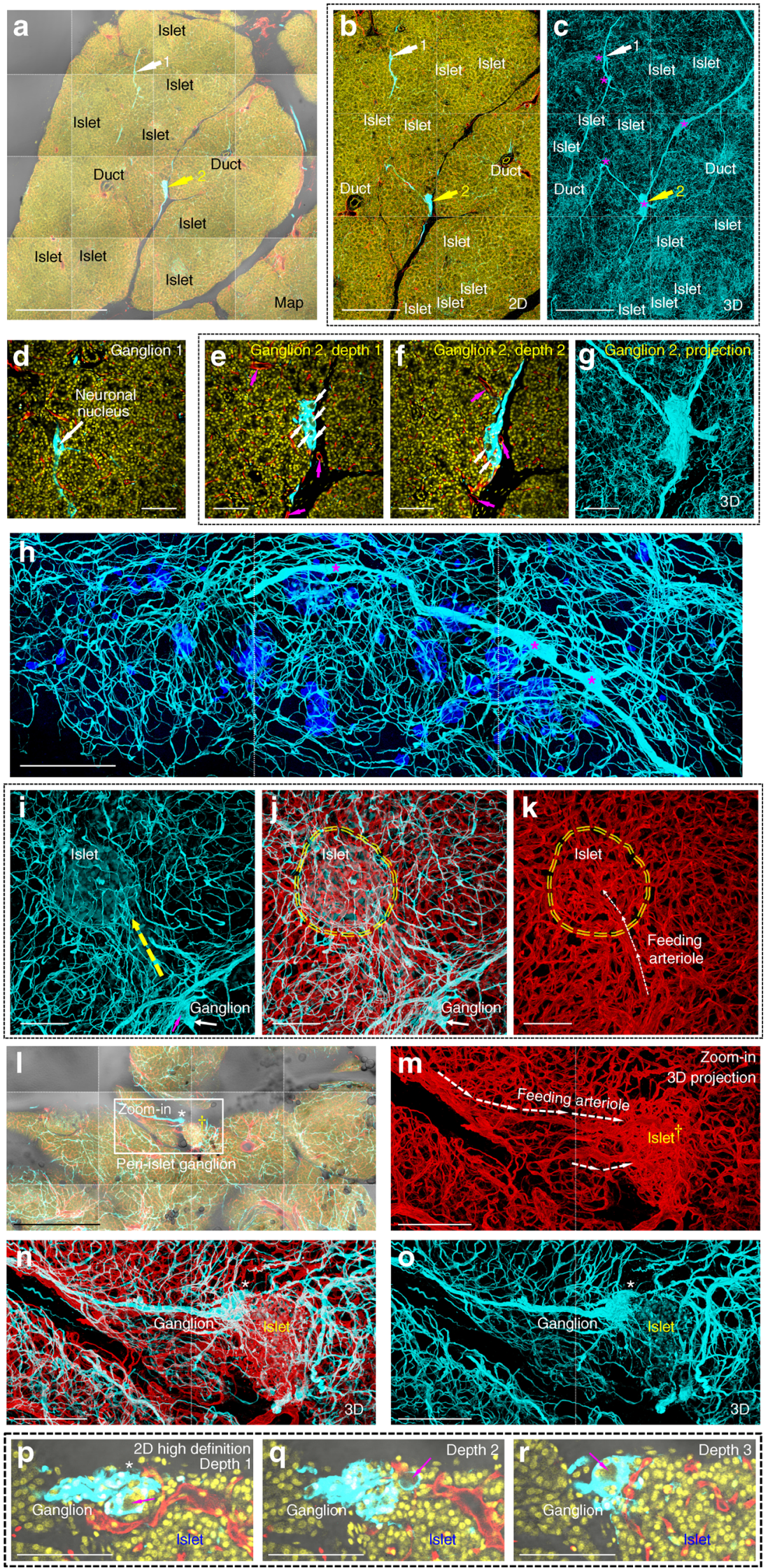
4 Fig. 7 Intra-pancreatic ganglia and islet-ganglionic association in human pancreas. (a-c) Gross view of glial marker S100B-labelled intra-pancreatic ganglia and glial network. (a) Tissue map (scale bar, $1 \mathrm{~mm})$. (b, c) 2D image and 3D projection of glial network and isletganglionic association (scale bars, $500 \mu \mathrm{m}$ ). Five ganglia are identified in (c) (magenta asterisks). Number 1 and 2 (arrows) are enlarged in (d-g). Note that islet cells show low-level S100B signals. Cyan, S100B; yellow, nuclei; red, CD31. (d-g) Two subgroups of intra-pancreatic ganglia. (d) The intra-parenchymal ganglia. (e-g) The peri-lobular ganglia. White arrows, neuronal nuclei; magenta arrows, supporting microvessels. Scale bars, $100 \mu \mathrm{m}$. (h) Islet-ganglionic association revealed by paired insulin and S100B staining (gross view). Blue, insulin; cyan, S100B; magenta asterisks, ganglia. Scale bar, $200 \mu \mathrm{m}$. Projection depth, $250 \mu \mathrm{m}$. (i-k) High-definition images of peri-islet ganglion and isletganglionic association. The three images were taken under the same view. In (i) and (k), dashed arrows show that glial fibres follow the feeding arteriole into the islet core. Oval, islet boundary. The peri-islet ganglion is labelled with solid arrows (magenta arrow in (i), soma). Scale bars, $100 \mu \mathrm{m}$. (I-r) Direct islet-ganglionic contact at the edge of parenchyma. (I) $2 \mathrm{D}$ pancreatic tissue map (donor 5, moderate fatty infiltration; asterisk, ganglion; dagger, islet; scale bar, $1 \mathrm{~mm})$. (m-o) 3D projection of peri-islet ganglion (scale bars, $200 \mu \mathrm{m}$ ); the ganglion is further enlarged in (p-r). (p-r) High-definition 2D images of peri-islet ganglion (three focal depths; scale bars, $100 \mu \mathrm{m}$ ). Magenta arrows, neuronal nucleus; cyan, S100B; yellow, nuclei; red, CD31

intra-parenchymal ganglia occurs. This increase could be attributed to the pancreatic fatty infiltration (Fig. 8f, g), in which adipocytes have been known to secrete nerve growth factors [21]. ESM Fig. 1 provides an additional example of the pancreatic microenvironment with the neuro-insular network in severe fatty infiltration. Overall, the 3D image data underscore an adaptive pancreatic nervous system that responds to infiltrated adipocytes.

\section{Discussion}

Because of the dispersed nature of blood vessels and nerves, islets and their associations with neurovascular networks cannot be easily observed using standard histology. In this research, the human pancreatic neurovascular networks are visualised via 3D panoramic histology with tissue clearing [10] to characterise the network architecture in a space continuum. Using the 3D image data, we identify a pancreatic neuro-insular network and islet-ganglionic associations in health and fatty infiltration. In the network, two subgroups of intra-pancreatic ganglia (the peri-lobular ganglia and the intra-parenchymal ganglia) are specified. Importantly, the ganglia appear to be reactive in pancreatic fatty infiltration, in which ganglionic enlargement and increases in nerve projections are identified. The results reflect a neurotrophic environment and an adaptive neural system around the infiltrated adipocytes. Finally, because humans and mice fundamentally differ in pancreatic fatty infiltration as well as for islet-acinar neurovascular association, caution must be exercised when using animal models to mimic human pancreatic microenvironments and neurovascular remodelling in obesity.

Although previous human islet imaging has suggested limited neural-islet associations, particularly a lack of parasympathetic nerves in the islet $[35,36]$, the improved imaging approach used here with tissue clearing has demonstrated that both neuronal and glial networks travel into the islet core (Figs 4, 5, 6, 7). In particular, the THlabelled sympathetic nerves and vAChT-labelled parasympathetic nerves reside in the immediate microenvironment of the islet cells (within an estimated $50 \mu \mathrm{m}$; Figs 4, 6 and ESM Video 2). These findings imply that the neurotransmitters released from the nerves can potentially reach the nearby endocrine cells via diffusion and/or spill-over into the microvessels to regulate downstream cellular activities. In addition, because the islet cells are coupled through gap junctions [38, 39], cells distal to nerves may be indirectly influenced via islet cell-cell communication.

The intra-pancreatic ganglia and the islet-ganglionic association identified in the human pancreas (Figs 5, 6, 7, 8 and ESM Fig. 1) suggest a potential integration of islets with the nervous system in the regulation of hormone secretion. This result and the neuro-insular association identified in mice [10] support a neurally mediated synchronisation of islet hormone secretion across species [40]. Importantly, in fatty infiltration, the formation of an adipose-ganglionic complex indicates that the pancreatic neural tissues are not bystanders in the remodelling of parenchyma (Fig. 8); they are targets affected by the infiltrated adipocytes, which could influence ganglionic activity.

The technical advance of tissue clearing of mouse and human gastrointestinal tissues $[10,37,41-43]$ has enabled us to develop 3D histology for visualisation of the pancreatic neuroinsular network. Using the transparent specimens, we applied both fluorescence and transmitted light microscopy (a dualoptical approach) to examine the pancreatic microstructure and neurovascular network. Transmitted light imaging is particularly important in examination of human pancreas because: (1) it identifies the infiltrated adipocytes; and (2) the transmitted light signals are the ground-truth tissue information to verify the fluorescence signals, thereby confirming the fluorescence-labelled 3D structures. Furthermore, in 3D histology, tissue information is continuously recorded to characterise novel pancreatic microstructures, such as adipose-ganglionic complexes (ESM Video 5), which cannot be portrayed via standard microtome-based histology. Overall, our dualoptical approach and the in-depth tissue information obtained circumvent uncertainties in delineating the intricate environment of the human pancreas. 

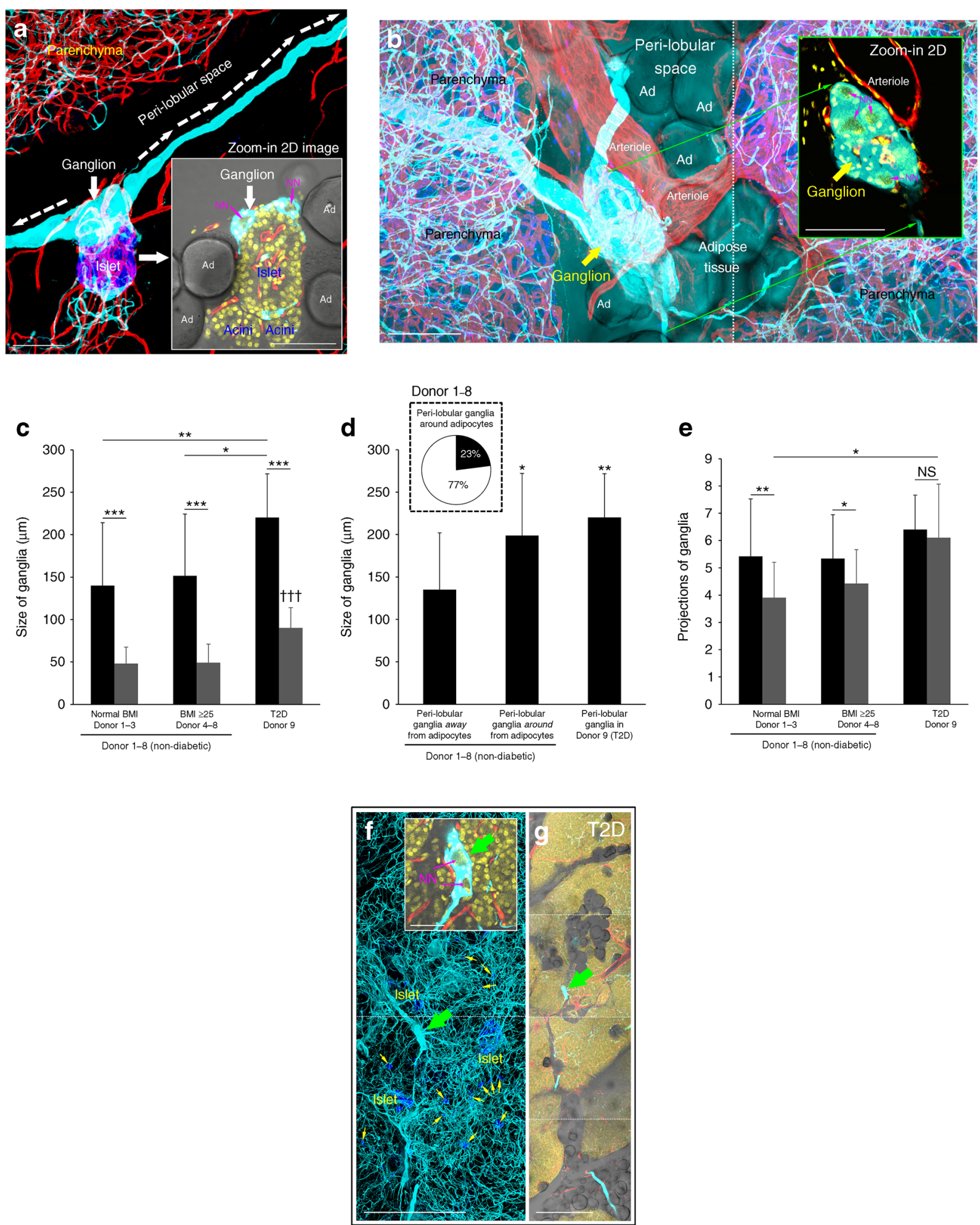

In conclusion, using cadaveric donor specimens we characterised the human pancreatic microenvironment and neuro-insular network in health and fatty infiltration. The human intra-pancreatic ganglia and islet neurovascular networks have not been systematically presented prior to this study due to a lack of 3D image data.
Here, the tissue maps and high-definition 3D images offer a global and integrated view of the human pancreatic environment. Our work will provide a technical and scientific basis for future research into an interaction among islets, adipocytes and the pancreatic nervous system in the progression of type 2 diabetes. 
4 Fig. 8 Qualitative and quantitative analyses of intra-pancreatic ganglia in health and fatty infiltration. (a, b) Peri-lobular fatty infiltration and adipose-ganglionic association. (a) Peri-islet ganglion and adiposeganglionic association (donor 6, moderate fatty infiltration). (b) Adipose-ganglionic association in severe fatty infiltration (donor 9; overlay of transmitted light and fluorescence image). Cyan, S100B; red, CD31; yellow, nuclei (inset); blue, insulin (a), nuclei and autofluorescence (b); magenta arrow, neuronal nucleus (NN; inset). Ad, adipocyte. (a, b) 3D projection. Inset: high-definition 2D image. Scale bars, $100 \mu \mathrm{m}$. (c) Size difference between peri-lobular (black bars) and intra-parenchymal (grey bars) ganglia in human pancreas. The difference is seen in specimens from both the non-diabetic and type 2 diabetic (T2D) donors. In the specimen from the T2D donor (donor 9), enlargement of peri-lobular and intra-parenchymal ganglia occurs compared with nondiabetic donors. $* p<0.05, * * p<0.01, * * * p<0.001 ;{ }^{\dagger \dagger} p<0.001$ vs both non-diabetic groups. (d) Enlargement of peri-lobular ganglia around adipocytes in fatty infiltration. $* p<0.05, * * p<0.01$ vs peri-lobular ganglia away $(>100 \mu \mathrm{m})$ from adipocytes. $n=50, n=15$ and $n=10$ ganglia from the 'away' (non-diabetic), 'around' (non-diabetic) and T2D conditions, respectively, were used in the analysis. All ten ganglia in the T2D condition are 'around' adipocytes. (e) Difference in number of nerve projections between peri-lobular (black bars) and intra-parenchymal (grey bars) ganglia. This feature is observed in the specimens from nondiabetic but not type 2 diabetic donors. $* p<0.05, * * p<0.01$. Number of peri-lobular and intra-parenchymal ganglia analysed in (c) and (e), respectively: donor $1, n=6 / n=12$; donor $2, n=6 / n=10$; donor 3 , $n=14 / n=10$; donor $4, n=5 / n=5$; donor $5, n=5 / n=12$; donor 6 , $n=13 / n=7$; donor $7, n=5 / n=7$; donor $8, n=11 / n=6$; and donor 9 , $n=10 / n=10$. Ganglia are pooled based on BMI and metabolic condition. (f, g) Intra-parenchymal, peri-islet ganglion in severe fatty infiltration. (f) 3D projection of peri-islet ganglion (inset: magnified examination). (g) 2D gross view of the ganglionic microenvironment. Green arrows indicate the same ganglion. Seven projections from the ganglion are identified. Blue, insulin; red, CD31; yellow, nuclei; yellow arrow, beta cell(s); magenta arrow (inset), neuronal nucleus. Scale bars: main image (f) and (g), $500 \mu \mathrm{m}$; inset (f), $50 \mu \mathrm{m}$

Acknowledgements We thank the support from the confocal imaging core in National Tsing Hua University (sponsored by the Ministry of Science and Technology [MOST]), Taiwan.

Data availability The images and datasets generated and/or analysed during the current study are available from the corresponding author on reasonable request.

Funding This work was supported in part by grants from the Taiwan National Health Research Institutes (NHRI-EX106-10524EI) and MOST (104-2314-B-007-002-MY2 and 104-2918-I-007-013) to SCT. LB is an advanced postdoctoral fellow of JDRF (3-APF-2014-183-A-N).

Duality of interest The authors declare that there is no duality of interest associated with this manuscript.

Contribution statement All authors contributed to the study concept and design. LB and MSG contributed to specimen acquisition. SCT, SJP and HJC contributed to 3D histology. SCT, LB, CNS, DWS, CEC and MSG contributed to 3D image presentation. SCT, LB and MSG contributed to drafting of the manuscript. All authors contributed to data analysis and interpretation of data, revised the manuscript critically for intellectual content, and approved the final version of the manuscript. SCT is the guarantor of this work and, as such, had full access to all the data in the study and takes responsibility for the integrity of the data and the accuracy of the data analysis.

\section{References}

1. Pirola L, Johnston AM, Van Obberghen E (2004) Modulation of insulin action. Diabetologia 47:170-184

2. Woods SC, Porte D Jr (1974) Neural control of the endocrine pancreas. Physiol Rev 54:596-619

3. Ahren B (2000) Autonomic regulation of islet hormone secretionimplications for health and disease. Diabetologia 43:393-410

4. Taborsky GJ Jr, Mundinger TO (2012) Minireview: the role of the autonomic nervous system in mediating the glucagon response to hypoglycemia. Endocrinology 153:1055-1062

5. Schwartz TW, Stenquist B, Olbe L (1979) Cephalic phase of pancreatic-polypeptide secretion studied by sham feeding in man. Scand J Gastroenterol 14:313-320

6. Teff KL (2010) Cephalic phase pancreatic polypeptide responses to liquid and solid stimuli in humans. Physiol Behav 99:317-323

7. Ahren B, Holst JJ (2001) The cephalic insulin response to meal ingestion in humans is dependent on both cholinergic and noncholinergic mechanisms and is important for postprandial glycemia. Diabetes 50:1030-1038

8. Teff KL (2011) How neural mediation of anticipatory and compensatory insulin release helps us tolerate food. Physiol Behav 103:44-50

9. Havel PJ, Mundinger TO, Taborsky GJ Jr (1996) Pancreatic sympathetic nerves contribute to increased glucagon secretion during severe hypoglycemia in dogs. Am J Phys 270:E20-E26

10. Tang SC, Shen CN, Lin PY, et al. (2017) Pancreatic neuro-insular network in young mice revealed by 3-D panoramic histology. Diabetologia doi:10.1007/s00125-017-4408-y

11. Dolensek J, Rupnik MS, Stozer A (2015) Structural similarities and differences between the human and the mouse pancreas. Islets 7 : e1024405

12. Smits MM, van Geenen EJ (2011) The clinical significance of pancreatic steatosis. Nat Rev Gastroenterol Hepatol 8:169-177

13. Lee JS, Kim SH, Jun DW et al (2009) Clinical implications of fatty pancreas: correlations between fatty pancreas and metabolic syndrome. World J Gastroenterol 15:1869-1875

14. Ou HY, Wang CY, Yang YC, Chen MF, Chang CJ (2013) The association between nonalcoholic fatty pancreas disease and diabetes. PLoS One 8:e62561

15. Wang CY, Ou HY, Chen MF, Chang TC, Chang CJ (2014) Enigmatic ectopic fat: prevalence of nonalcoholic fatty pancreas disease and its associated factors in a Chinese population. J Am Heart Assoc 3:e000297

16. Lingvay I, Esser V, Legendre JL et al (2009) Noninvasive quantification of pancreatic fat in humans. J Clin Endocrinol Metab 94: 4070-4076

17. Sorisky A (1999) From preadipocyte to adipocyte: differentiationdirected signals of insulin from the cell surface to the nucleus. Crit Rev Clin Lab Sci 36:1-34

18. Klemm DJ, Leitner JW, Watson P et al (2001) Insulin-induced adipocyte differentiation. Activation of CREB rescues adipogenesis from the arrest caused by inhibition of prenylation. J Biol Chem 276:28430-28435

19. Geloen A, Collet AJ, Guay G, Bukowiecki LJ (1989) Insulin stimulates in vivo cell proliferation in white adipose tissue. Am J Phys 256: $\mathrm{C} 190-\mathrm{C} 196$

20. Cao Y (2007) Angiogenesis modulates adipogenesis and obesity. J Clin Invest 117:2362-2368

21. Peeraully MR, Jenkins JR, Trayhurn P (2004) NGF gene expression and secretion in white adipose tissue: regulation in 3T3-L1 
adipocytes by hormones and inflammatory cytokines. Am J Physiol Endocrinol Metab 287:E331-E339

22. Fu YY, Tang SC (2010) At the movies: 3-dimensional technology and gastrointestinal histology. Gastroenterology 139:1100-1105

23. Chien HJ, Peng SJ, Hua TE, Kuo CH, Juang JH, Tang SC (2016) 3$\mathrm{D}$ imaging of islets in obesity: formation of the islet-duct complex and neurovascular remodeling in young hyperphagic mice. Int $\mathbf{J}$ Obes 40:685-697

24. Winzell MS, Ahren B (2004) The high-fat diet-fed mouse: a model for studying mechanisms and treatment of impaired glucose tolerance and type 2 diabetes. Diabetes 53(Suppl 3):S215-S219

25. Fu YY, Lin CW, Enikolopov G, Sibley E, Chiang AS, Tang SC (2009) Microtome-free 3-dimensional confocal imaging method for visualization of mouse intestine with subcellular-level resolution. Gastroenterology 137:453-465

26. Juang JH, Peng SJ, Kuo CH, Tang SC (2014) Three-dimensional islet graft histology: panoramic imaging of neural plasticity in sympathetic reinnervation of transplanted islets under the kidney capsule. Am J Physiol Endocrinol Metab 306:E559-E570

27. Genina EA, Bashkatov AN, Tuchin VV (2010) Tissue optical immersion clearing. Expert Rev Med Devices 7:825-842

28. Bosco D, Armanet M, Morel P et al (2010) Unique arrangement of alpha- and beta-cells in human islets of Langerhans. Diabetes 59: $1202-1210$

29. Cabrera O, Berman DM, Kenyon NS, Ricordi C, Berggren PO, Caicedo A (2006) The unique cytoarchitecture of human pancreatic islets has implications for islet cell function. Proc Natl Acad Sci U S A 103:2334-2339

30. Bonner-Weir S, Orci L (1982) New perspectives on the microvasculature of the islets of Langerhans in the rat. Diabetes 31:883-889

31. Brissova M, Shostak A, Fligner CL et al (2015) Human islets have fewer blood vessels than mouse islets and the density of islet vascular structures is increased in type 2 diabetes. J Histochem Cytochem 63:637-645

32. Cohrs CM, Chen C, Jahn SR et al (2017) Vessel network architecture of adult human islets promotes distinct cell-cell interactions in situ and is altered after transplantation. Endocrinology 158:13731385

33. Chiu YC, Hua TE, Fu YY, Pasricha PJ, Tang SC (2012) 3-D imaging and illustration of the perfusive mouse islet sympathetic innervation and its remodelling in injury. Diabetologia 55:3252-3261

34. Portela-Gomes GM, Hacker GW, Weitgasser R (2004) Neuroendocrine cell markers for pancreatic islets and tumors. Appl Immunohistochem Mol Morphol 12:183-192

35. Rodriguez-Diaz R, Abdulreda MH, Formoso AL et al (2011) Innervation patterns of autonomic axons in the human endocrine pancreas. Cell Metab 14:45-54

36. Rodriguez-Diaz R, Dando R, Jacques-Silva MC et al (2011) Alpha cells secrete acetylcholine as a non-neuronal paracrine signal priming beta cell function in humans. Nat Med 17:888-892

37. Liu YA, Chung YC, Pan ST et al (2013) 3-D imaging, illustration, and quantitation of enteric glial network in transparent human colon mucosa. Neurogastroenterol Motil 25:e324-e338

38. Serre-Beinier V, Bosco D, Zulianello L et al (2009) Cx36 makes channels coupling human pancreatic beta-cells, and correlates with insulin expression. Hum Mol Genet 18:428-439

39. Rutter GA, Hodson DJ (2013) Minireview: intra-islet regulation of insulin secretion in humans. Mol Endocrinol 27:1984-1995

40. Satin LS, Butler PC, Ha J, Sherman AS (2015) Pulsatile insulin secretion, impaired glucose tolerance and type 2 diabetes. Mol Asp Med 42:61-77

41. Smith K (2011) Neurogastroenterology: improving 3D imaging of the enteric nervous system. Nat Rev Gastroenterol Hepatol 8:600

42. Liu YA, Chung YC, Pan ST, Pasricha PJ, Tang SC (2015) Perivascular interstitial cells of Cajal in human colon. $\mathrm{CMGH}$ 144:S372-S372

43. Lin PY, Peng SJ, Shen CN, Pasricha PJ, Tang SC (2016) PanINassociated pericyte, glial, and islet remodeling in mice revealed by 3D pancreatic duct lesion histology. Am J Physiol Gastrointest Liver Physiol 311:G412-G422 